\title{
Functional morphology of the raptorial forelegs in Mantispa styriaca (Insecta: Neuroptera)
}

\author{
Sebastian Büsse ${ }^{1}$ (D) Fabian Bäumler ${ }^{1}$ (D) $\cdot$ Stanislav N. Gorb ${ }^{1}$ (D)
}

Received: 14 September 2020 / Revised: 26 March 2021 / Accepted: 30 March 2021 / Published online: 12 April 2021

(c) The Author(s) 2021

\begin{abstract}
The insect leg is a multifunctional device, varying tremendously in form and function within Insecta: from a common walking leg, to burrowing, swimming or jumping devices, up to spinning apparatuses or tools for prey capturing. Raptorial forelegs, as predatory striking and grasping devices, represent a prominent example for convergent evolution within insects showing strong morphological and behavioural adaptations for a lifestyle as an ambush predator. However, apart from praying mantises (Mantodea) — the most prominent example of this lifestyle — the knowledge on morphology, anatomy, and the functionality of insect raptorial forelegs, in general, is scarce. Here, we show a detailed morphological description of raptorial forelegs of Mantispa styriaca (Neuroptera), including musculature and the material composition in their cuticle; further, we will discuss the mechanism of the predatory strike. We could confirm all 15 muscles previously described for mantis lacewings, regarding extrinsic and intrinsic musculature, expanding it for one important new muscle-M24c. Combining the information from all of our results, we were able to identify a possible catapult mechanism (latch-mediated spring actuation system) as a driving force of the predatory strike, never proposed for mantis lacewings before. Our results lead to a better understanding of the biomechanical aspects of the predatory strike in Mantispidae. This study further represents a starting point for a comprehensive biomechanical investigation of the convergently evolved raptorial forelegs in insects.
\end{abstract}

Keywords Mantis fly $\cdot$ Lacewing $\cdot$ Striking legs $\cdot$ Predatory strike $\cdot$ Catapult mechanism $\cdot$ Spring actuation $\cdot$ Insect cuticle

\section{Introduction}

The insect legs are locomotory appendages in the first place. However, they can vary greatly in functional abilities and morphological attributes: for example, bees store pollen in a specialized basket at their hind legs (Martins et al. 2014), webspinners spin their homes with a spinning apparatus located in their front feet (Büsse et al. 2015), and cicadas jump using their enlarged hind legs (Gorb 2004). Nevertheless, all these insects still utilize their legs for walking as well. Here, another example for this multifunctionality is presented, the raptorial forelegs. These prehensile legs are used for prey capturing and can most commonly be divided into two different types within insects: chelate and subchelate raptorial forelegs legs (compare Fig. 1 in Weirauch et al.

Sebastian Büsse

sbuesse@zoologie.uni-kiel.de

1 Department of Functional Morphology and Biomechanics, Institute of Zoology, Kiel University, Am Botanischen Garten 9, 24118 Kiel, Germany
2011). The chelate type — best known as 'crab claws' —is in insects present in some Reduviidae (Hemiptera; Weirauch et al. 2011). The sub-chelate type raptorial forelegs are best known for Mantodea (see Roy 1999; Wieland 2013 or Brannoch et al. 2017). However, they can be found in other insect groups, such as Hemiptera, Diptera and Neuroptera as well, representing a prominent example of convergent evolution (Brémond 1974; Simpson 1975; Poivre 1976; CastroHuertas et al. 2019). All these insects are ambush predators, showing strong morphological adaptations regarding the use of their forelegs as predatory striking and grasping devices (Ferris 1940; Ulrich 1965; Matsuda 1970; Loxton and Nicholls 1979; Frantsevich 1998). At first glance, praying mantises and mantis lacewings (Neuroptera: Mantispidae) share numerous morphological similarities (Ferris 1940; Ulrich 1965; Frantsevich 1998): a very movable, and often elongated, prothorax to strike for large distances (Frantsevich 1998), strong leg muscles to perform a fast predatory strike (Ulrich 1965; Gray and Mill 1983, 1985), as well as elongated and spined leg articles to hold the prey (Loxton and Nicholls 1979; Roy 1999; Wieland 2013). However, 


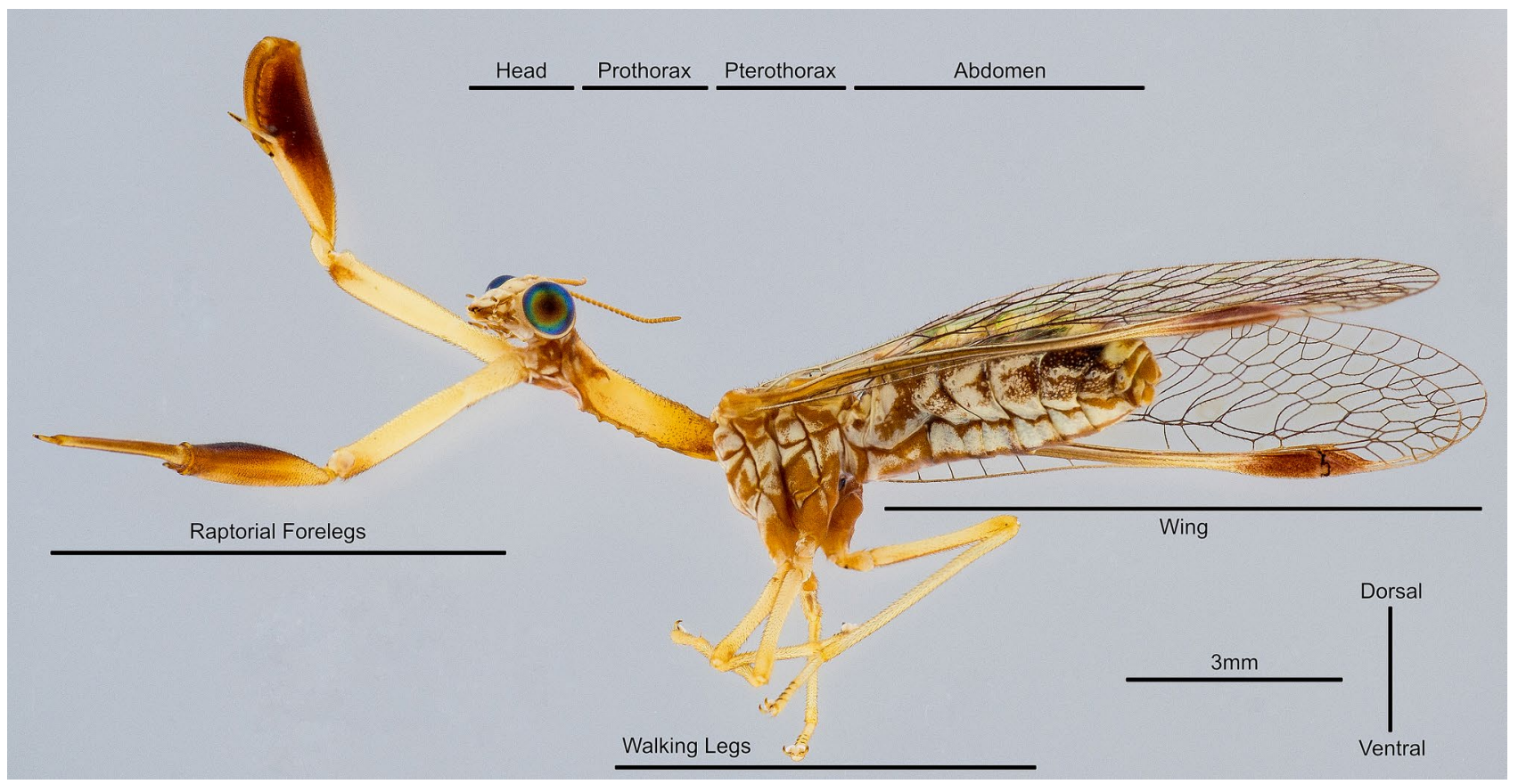

Fig. 1 Lateral view of Mantispa styriaca

Mantispidae in general, show some very unique behavioural and morphological adaptations (Aspöck et al. 1980; Aspöck and Aspöck 1994, 2007; Redborg 1998). Representatives of the sub-group Mantispinae, for example, are spider associated parasites (Kaston 1938; Redborg and MacLeod 1983; Rice 1986; Redborg 1998; Aspöck and Aspöck 2007; Haug et al. 2018); within this context one of the few detailed studies on functional leg morphology in neuropterans was conducted, describing the highly specialised leg of the first larval instar of a representative (Jandausch et al. 2018).

The adult mantis lacewing is a classic sit-and-wait ambush predator (Boyden 1983), highly specialised to a predatory lifestyle (Fig. 1), as described above. The prothorax is elongated and flexibly connected to the following mesothorax (Ferris 1940; Ulrich 1965). The foreleg suspension is adapted for reliable aiming and controlling of fast and powerful strikes. Furthermore, it must provide enough versatility to hold and handle the prey (Frantsevich 1998). The leg articles-especially femur, tibia, and tarsus-are strongly modified: the femur is thickened and thorned, the tibia is blade-like and the tarsus forms a rather immobile elongation of the tibia (Ferris 1940; Ulrich 1965). Morphological differences, especially in the occurrence of femoral and tibial spines, in fossil and extant representatives of the sub-groups of Mantispidae are multifarious and allow for evolutionary and/or phylogenetic implications (Shi et al. 2015; Pérez-de la Fuente and Peñalver 2019). But details on the anatomy of the raptorial foreleg's (Frantsevich 1998) as well as functional implications are rare.
The material composition of the raptorial foreleg's cuticle of any insect, was, to our knowledge, never investigated. Yet, Loxton and Nicholls (1979) indicated differences within the cuticle in Mantodea, due to either heavily sclerotized or thin and flexible cuticle at the base of femoral spines, that needs further investigation. However, the relevance of the material composition in a functional and/or biomechanical context, was shown in a number of studies for a variety of character systems in insects like: mouthparts (Büsse and Gorb 2018), thorax (Bäumler and Büsse 2019) or abdomen (Willkommen et al. 2015), but most often for wings (Gorb 1999; Haas et al., 2000; Appel and Gorb 2011; Rajabi et al. 2016) and legs (Peisker et al. 2013; Jandausch et al. 2018; Petersen et al. 2018; Büsse et al. 2019a, b). The main purpose of the study is therefore, to present a detailed description of the raptorial forelegs of Mantispa styriaca (Poda, 1761), including extrinsic and intrinsic musculature as well as the material composition of their cuticle. Based on these data, we propose a springactuated mechanism (catapult mechanism) as driving force for the predatory strike, never suggested for mantis lacewings before. This results in a better understanding of biomechanical aspects of the predatory strike in Mantispidae, additionally allowing for a comparison to that of the Mantodea. This study represents a starting point to investigate the convergent evolution of raptorial forelegs in insects, giving new insights from a biomechanically very interesting, but often overlooked character system. 


\section{Materials and methods}

Adult Mantispa styriaca (Fig. 1), from the collection of the Museum of Natural History Vienna (Austria), were used. The specimens were stored in $70-80 \%$ ethanol. Prior to scanning, both in micro-computed tomography $(\mu \mathrm{CT})$ and scanning electron microscopy (SEM), the samples were dehydrated in an ascending ethanol series and dried at the critical point using a fully automatic Leica EM CPD300 (Leica, Wetzlar, Germany) CPD system.

High resolution X-ray tomography $(\mu \mathrm{CT})$ was carried out using a SkyScan 1172 desktop $\mu$ CT scanner (Bruker micro-CT, Kontich, Belgium) at $40 \mathrm{kV}$ and $250 \mu \mathrm{A}$, with images taken over a full $360^{\circ}$ rotation (step size: $0.25^{\circ}$ ). The segmentation of the data was done in Amira 6.2 (Thermo Fisher Scientific, Waltham, USA) and for visualization, the open source 3D creation suite Blender 2.82a (Blender Foundation, Amsterdam, Netherlands) was used.

For scanning electron microscopy (SEM), the dried samples were sputter-coated with gold-palladium $(10 \mathrm{~nm}$ thickness; Leica Bal-TEC SCD500, Leica Camera AG, Wetzlar, Germany). Afterwards, the samples were mounted on a rotatable sample holder (Pohl 2010) and examined in a Hitachi TM3000 (Hitachi Ltd. Corporation, Tokyo, Japan) scanning electron microscope at an accelerating voltage of $15 \mathrm{kV}$.

For confocal laser scanning microscopy (CLSM), the legs were dissected and embedded in glycerine $(99.9 \%)$ on a glass slide and covered with a high-precision cover slip prior to scanning. For CLSM visualisation, a Zeiss LSM 700 (Carl Zeiss AG, Jena, Germany) was used. Four laser lines (wavelengths of 405, 488, 555 and $639 \mathrm{~nm}$ ) and four emission filters (BP420-480, LP490, LP560, LP640 nm) were used to visualize the autofluorescence of the cuticle (cf. Fig. 1 in Büsse and Gorb 2018). Maximum intensity projections were combined using ZEN2008 software (Carl Zeiss Microscopy AG, Jena, Germany). For more information on using CLSM to determine the material properties of the insect cuticle, we refer to Michels and Gorb (2012). The maximum intensity projections of the CLSM analysis, result in visualization of combined autofluorescence signals in every single pixel, providing information about the presence of various components within the cuticle with different material properties (Andersen 1979; Vincent 2002; Michels and Gorb 2012). It is therefore possible, to estimate the material composition of the analysed cuticle. The following colour code can be assigned: (1) red autofluorescence - stiff sclerotized cuticle; (2) blue, green and red combined (resulting in pink, brownish, yellow, and green autofluorescence within the overlay)stiff but more flexible cuticle, if compared with the previous one, often chitin dominated; (3) characteristic blue autofluorescence- rubber-like cuticle with likely high proportion of resilin. The results of the CLSM analyses described in the following section allow for a qualitative assessment only and does not represent a quantitative measurement.

For stacked photography, we used a custom-made 3D-printed illumination dome system (Bäumler et al. 2020) and an Olympus OMD 10mkII digital camera (Olympus K.K., Tokyo, Japan), equipped with a Leica $45 \mathrm{~mm}$ macro lens (Leica Camera AG, Wetzlar, Germany). All images were subsequently processed in Affinity Photo and Affinity Designer (Serif Ltd, Nottingham, United Kingdom).

Extrinsic leg musculature terminology follows the nomenclature introduced by Friedrich and Beutel (2008), intrinsic leg musculature terminology follows the nomenclature used in Jandausch et al. (2018). Additionally, all terms regarding the outer morphology of the raptorial foreleg follow the nomenclature introduced by Wieland (2013) for Mantodea. The plane of orientation for the legs of $M$. styriaca are assigned, as if the forelegs were orientated as a cursorial insect leg, i.e. stretched aside (in congruence with Gray and Mill 1985; Wieland 2013). Newly introduced terms not found in the literature are defined at their first appearance in the text and put in quotation marks ('...').

\section{Results}

\section{External leg morphology}

In general, the sub-chelate type of raptorial forelegs is featured. The coxa of the raptorial foreleg is strongly elongated and the foreleg suspension ('coxo-thoracic joint'-ctj) consists of large membranous areas (Fig. 2), reaching further posterior in ventral than in dorsal direction. Furthermore, the fronto-coxa shows one articulatory point with the pleural process, and the pleural condyle is connected via a ball and socket type joint with the coxa (Frantsevich 1998). The coxa is covered with a vestiture of small setae along the dorsal, and more strongly along the ventral area (Fig. 2). Here, a distinct posterodorsal 'rabbet', consisting of a dorsal and a posterior notch; forming a rabbet-like slot is present on the surface (Figs. 1, 2).

A small number of setae can be found on the trochanter. The trochanter is linked to the femur via a bowl-shaped dorsal oriented joint, becoming narrower towards the pointy distal end. The femur is almost triangular, with a wider and thicker area in the middle. It shows a 'posteroventral carina' (pvc), equipped with a median row of spines (referred to as 'anteroventral spines'-avs; Fig. 3), running from the widest point in the middle up to the proximal end. The most prominent character is a large, at the base bended, spine on the anteroventral side - the 'subbasal spine' (sbs; introduced 


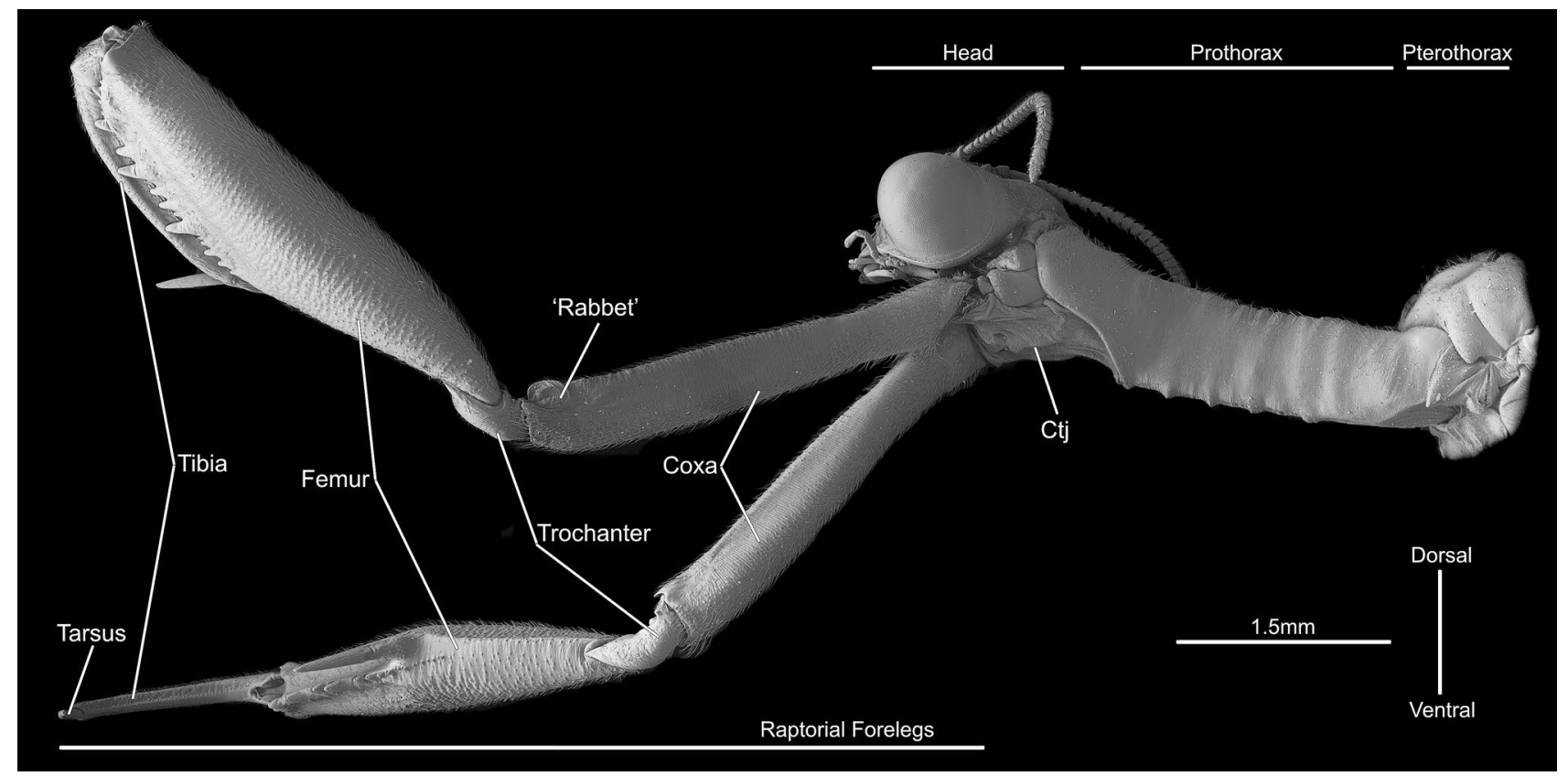

Fig. 2 Scanning electron microscopy micrograph of Mantispa styriaca, ventrolateral view of head, prothorax and raptorial forelegs. Ctj coxothoracic joint

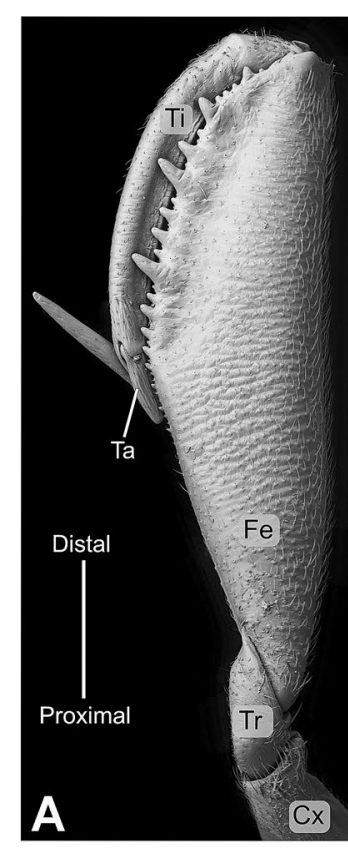

Fig. 3 Scanning electron microscopy micrographs of Mantispa styriaca, forelegs. a, $\mathbf{c}$ anterior and posterior view of closed raptorial foreleg; $\mathbf{b}, \mathbf{d}$ anterior and posterior view of open raptorial foreleg; $\mathbf{e}$ ventral view of femur. As area of setae, Avs anteroventral spines, Btn

from Willmann 1990 as major spine) —at the base of the carina, approximately in the middle of the femur (Fig. 3). A vestiture of small setae is almost completely covering the femur. This coverage is dense at the base of the femur, on

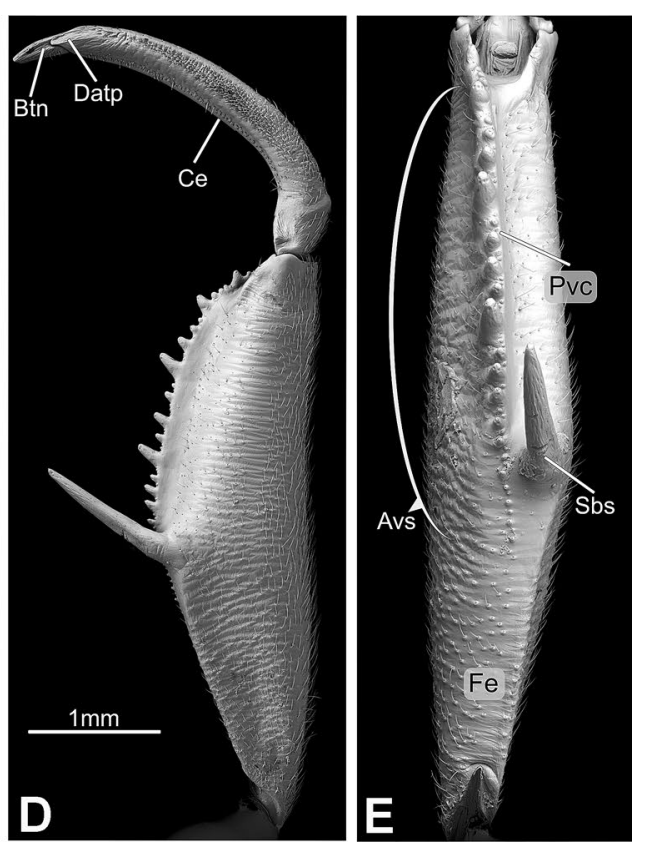

basitarsal notch, $C e$ cutting edge, $C x$ coxa, Datp distal anterotibial protrusion, Dptp distal posterotibial protrusion, $F e$ femur, $P v c$ posteroventral carina, $S b s$ subbasal spine, $T a$ tarsus, $T i$ tibia, $T r$ trochanter

the dorsal, and the anterior side (except on the mentioned carina; Fig. 3). On the anterior side and in a close radius around the proximal area of the carina, only a small quantity of setae is developed (Fig. 3). The tibial base is widened and 
reinforced, before bending further distal into a sickle-shape, with a distinct blade-like 'cutting edge' (ce) on the ventral side (Figs. 2, 3). A patch of setae can be found on the dorsal base, a few setae along the dorsal side and at the base of the cutting edge. Furthermore, a long triangular-shaped area, densely packed with small and thickened setae is located on the anterior side of the tibia.

Expanding and forming the tip of the sickle-shape, the first tarsal article is an elongation of the tibial sickle. Additionally, the distal tip of the tibia forms a smaller posterior and larger anterior thorn-like protrusion-'distal posterotibial protrusion' (dptp) and 'distal anterotibial protrusion' (datp). The latter (datp) placed into a 'basitarsal notch' (btn) on the anterior side (Fig. 3).

\section{Material composition}

The most prominent parts of the raptorial foreleg (femur, tibia, and tarsus), exhibit a mixture of very different material properties (Fig. 4). The anterior area of the femur is strongly sclerotized (Fig. 4a), whereas the posterior area is a patchwork of resilin-dominated and chitinous parts, showing a median area of resilin-dominated cuticle ('femoral posterior pad'-fpp; Fig. 4e). At the base of the femur (anterior view; Fig. 4a), a v-shaped, strongly resilin-dominated area stretches dorsally ('femoral dorsobasal pad'-fdbp; approx. $1 / 3$ the length of the femur) and ventrally ('femoral ventrobasal pad'-fvbp) up to the subbasal spine (approx. 1/2 the length of the femur) (Fig. 4a) - stretching onto the subbasal spine ('subbasal spine pad' - sbsp) facing proximal (Fig. 4b). In contrast, the distal face of the subbasal spine is strongly sclerotised (especially at the base), so is the tip. The anteroventral carina with its spines, is embedded in sclerotised cuticle anteriorly and chitinous cuticle posteriorly; the carina itself is also strongly sclerotised (Fig. 4a, e). The sickle-shaped tibia is more sclerotised on its anterior face and shows a line of resilin-dominated cuticle running from proximal to distal on the posterior face ('tibial posterior pad'-tpp; Fig. 4e). Additionally, the base of the tibia is slightly thickened and chitinous, with one anteriorly located resilin-dominated pad ('tibial anterobasal pad'tabp; Fig. 4d) and one posteriorly oriented at the distal end (Fig. 4c). The mentioned cutting edge of the tibia is strongly sclerotised. At the distal tip of the tibia, a sclerotised protrusion-'distal anterior protrusion' (dap)—on the anterior side is developed.

The entire setae on the femur and tibia show no auto-fluorescence, appearing black in the CLSM maximum intensity projection (Fig. 4); which is presumably due to the presence of melanin in the cuticle.

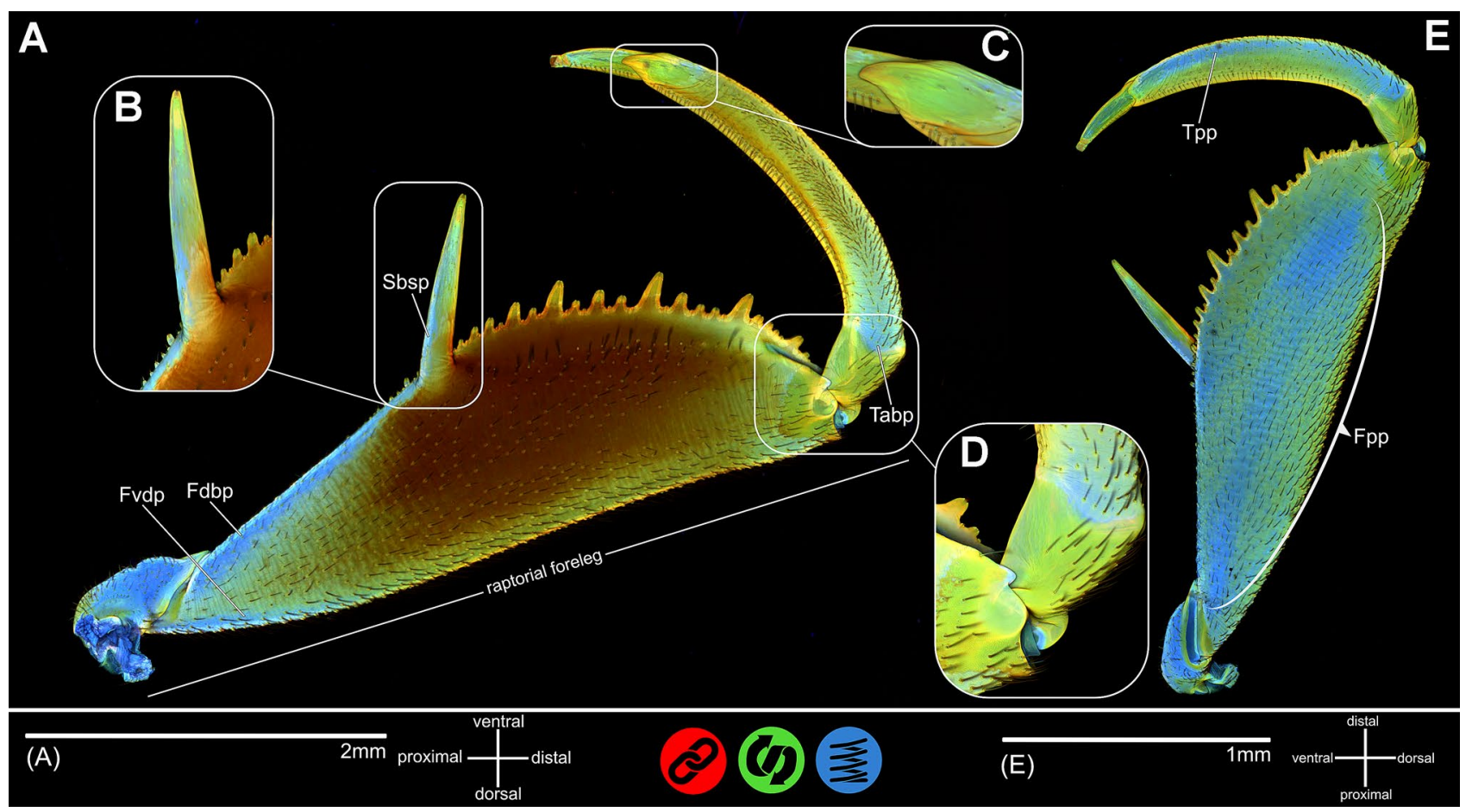

Fig. 4 Maximum intensity projection (CLSM) of the raptorial foreleg of Mantispa styriaca. a anterior view; b enlarged view of subbasal spine, $\mathbf{C}$ enlarged view of the distal tibial protrusion, $\mathbf{d}$ enlarged view of the widened tibial base, e posterior view. $F d b p$ femoral dorsobasal pad, $F v d p$ femoral ventrobasal pad, Fpp femoral posterior pad, $S b s p$ subbasal spine pad, Tadp tibial anterobasal pad, Tpp tibial posterior pad 


\section{Musculature}

In all following descriptions, the non-moving end of a muscle will be referred to as a point of origin $(\mathrm{O})$ and its moving end will be referred to as a point of insertion (I). All names for muscles follow the nomenclature introduced by Beutel et al. (2014), supplemented with information from Jandausch et al. (2018). Muscles marked with an asterisk are newly described.

\section{Extrinsic musculature (Fig. 5)}

Dorso-ventral musculature Idvm15-M. pronoto-trochantinocoxalis

O: Anterior part of pronotum.

I: At anterior procoxal rim next to Ipcm5.

Pleuro-coxal musculature Ipcm4-M. propleuro-coxalis superior

O: Anterodorsal area of propleura.

I: Lateral at procoxal articulation.

Ipcm5-M. propleuro-coxalis inferior

O: Posteroventral face of propleural apodeme.

I: At anterior procoxal rim next to Idvm 15 .

Ipcm6-propleuro-coxalis posterior

O: Anteroventral face of propleural apodeme.

I: Posterolateral procoxal rim.
Sterno-coxal musculature Iscm1-M. profurca-coxalis anterior

O: Lateral face of profurca stem.

I: Median, at proximal procoxal wall.

Iscm3-M. profurca-coxalis medialis

O: Lateral face of profurca.

I: Anteromesal procoxal rim.

\section{Intrinsic musculature (Fig. 5)}

Coxa:

ctm-Coxal-trochanteral muscle

O: Posterolateral procoxal rim.

I: Ventral trochanteral protrusion, dorsal of the insertion of M20 and M21.

M20-M. pleura-trochanteralis

O: Anteroventral wall of distal coxal half.

I: Ventral trochanteral protrusion, anteroventral of $\mathrm{ctm}$.

M21-M. coxa-trochanteralis medialis

O: Posteroventral wall of distal coxal half.

I: Ventral trochanteral protrusion, posteroventral of $\mathrm{ctm}$.

M22-M. coxa-trochanteralis lateralis

O: Three bundles of muscles, at anterior and posterior wall of distal half of coxa and at dorsal area of proximal coxal rim.

I: Dorsal, at protrusion at trochanteral rim.

Trochanter:

M23-M. reductor femoris

O: Ventral area of proximal end of trochanter.

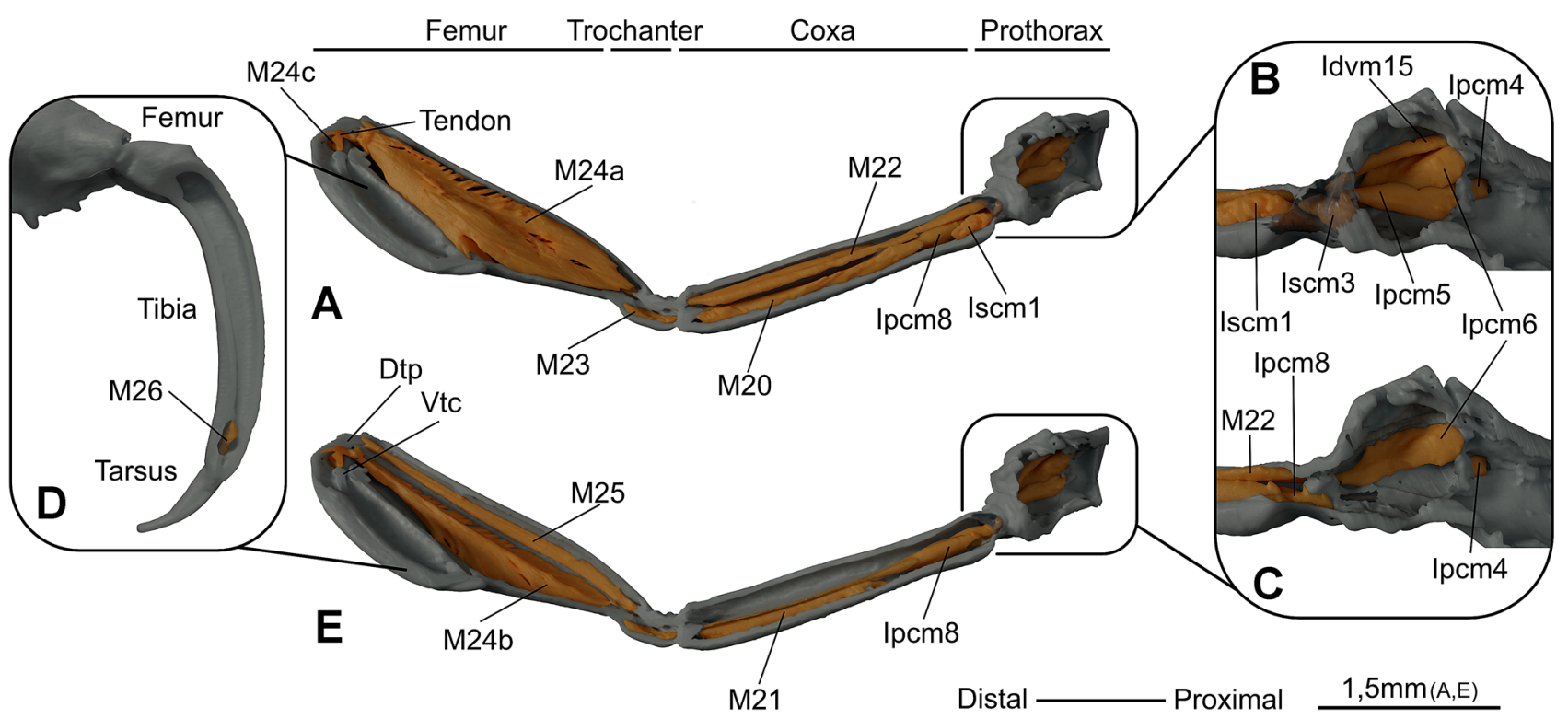

Fig. 5 Musculature of Mantispa styriaca's raptorial foreleg. Threedimensional visualisation from $\mu \mathrm{CT}$ data, lateral view. At certain areas, the cuticle is displayed transparent, to get a better view of the musculature. a, e, d intrinsic musculature; b, c extrinsic musculature; a, b inner layer; c, e outer layer. Dtp dorsal tibial protrusion, $M$ muscle, $P c m$ pleurocoxal musculature, $\mathrm{Scm}$ sternocoxal musculature, Vtc ventral tibial complex 
I: Ventral, at most proximal end of femur.

Femur:

M24a-dorsal median und ventral

M. depressor tibiae a.

O: Anterodorsal to ventral area of proximal $2 / 3$ of femur.

I: Together with M24b with a tendon at the enlarged tibial basis.

M24b-dorsal lateral und ventral

M. depressor tibiae $b$.

O: Posterio-dorsal to anteroventral area of proximal $2 / 3$ of femur.

I: Together with M24a with a tendon at the enlarged tibial basis.

M25-M. levator tibiae

O: On dorsal area at most proximal region of femur.

I: Dorsal, on proximal rim of tibia.

Tibia:

M26-M. depressor pretarsi

O: Dorsal area of distal tibia.

I: Probably on tendon to claw.

M24c*-M. depressor tibiae O: With two strains on the ventrolateral and ventromedian base of the tibia. I: At the tendon, originating from muscle M24a \& M24b.

\section{Discussion}

As previously mentioned, mantis lacewings share many morphological similarities with praying mantises, while also having particular separating morphological features. Interestingly, the raptorial forelegs of mantis lacewings are highly flexible in the coxo-thoracic, but also the coxotrochanteral joint. As already described by Frantsevich (1998), this is supported by the large membranous area of the coxothoracic joint (Figs. 1, 2)—resembling a multiaxial ball-and-socketlike joint. This flexibility allows for various movements like grooming (Lequet 2018: 0:50-0:58), handling prey (Lequet 2018: 2:10-2:18; Granier 2019: 0:40-0:50), grasping or walking (Frantsevitch 1998). The trochanter appears to be almost completely fused with the femur (Fig. 2), restricting any movement between those two parts to a minimum and forming a trochanter-femur complex.

In contrast, the joint between coxa and trochanter allows for a high degree of freedom. While handling prey (Lequet 2018: 2:10-2:18; Granier 2019: 0:41-0:55), a rotation of the coxotrochanteral joint of about $35^{\circ}$ can be observed. At the distal end of the coxa, the rabbet is formed, consisting of one dorsal and one posterior notch forming a slot (Fig. 2). It likely stabilizes the trochanter-coxa joint in resting position, by limiting the possible degrees of freedom to a minimum in the anterior and posterior direction (Lequet 2018: 0:00-0:10). Typically, mantis lacewings using their mesoand metathoracic legs for walking only, here stabilizing and keeping the forelegs in a safe position is of major importance (Lequet 2018). The mentioned high degree of flexibility is further supported, by the arrangement of extrinsic muscle bundles (Fig. 5), as previously discussed by Frantsevitch (1998). As described in Jandausch et al. (2018) and found in the present study as well, the muscle M22 consists of 3 muscle bundles - one originating anterior, one posterior and one in the center of the proximal coxa-all inserting at the same region of the trochanteral rim (Fig. 5). This separation in three bundles may lead to different hypothetical rotational movements of the trochanter-femur complex: (i) central bundle-general depression (ii) anterior and posterior bundles-adjustments of the respective spatial orientation. Here, handling of prey while feeding may be improved significantly. Muscles M20, M21 and CTM are functioning as antagonists to M22, elevating the trochanter-femur complex (Fig. 5).

In contrast to mantis lacewings, praying mantises show more than twice as many muscles that interact between thorax, coxa and trochanter (Gray and Mill 1985; Frantsevitch 1998). Although, the reasons are not clear yet, a few possibilities are already discussible. A higher number of different muscles could facilitate adjustments of the predatory strike to varying situations, to might improve hunting success. Furthermore, it is probably necessary to use the predatory forelegs for additional purposes, such as walking and increased load-carrying compared to M. styriaca.

Also functioning in a similar way to praying mantises, particular differences of the predatory strike and the raptorial forelegs are noticeable (Granier 2019: 1:28-1:35; Jo Alwood 2016: 1:20-1:30; Josephs 2017: 0:00-0:05). In contrast to praying mantises (Brannoch et al. 2017), M. styriaca has no distal tibial spur; as this seems also to be true for the fossil mantodean lineage (Dittmann et al. 2015; Hörnig et al. 2017). In modern praying mantises, this tibial spur is described to cooperate with the discoidal spine(s), to lock the prey item when the tibia closes on the femur (Fig. 2 in Loxton and Nicholls 1979). Furthermore, the discoidal spine is described as surrounded by an area of soft cuticle and therefore, movable in distal direction to the femur (Fig. 1 in Loxton and Nicholls 1979). When capturing prey, those two spines work as a lever and ratchet system, as described for praying mantises in Loxton (Fig. 5 in Loxton and Nicholls 1979). The same mechanism is conceivable, in M. styriaca's raptorial forelegs. As the lock-like structure, formed by the distal tibial protrusions (dptp, datp in Fig. 3b, d) together with the basitarsal notch, is noticeably sclerotized (Fig. 4c, e), it expands and elongates the sickle-shape of the tibia. Likely it has a similar purpose as the tibial spur in praying mantises. Additionally, the material composition of $M$. styriaca's subbasal spine supports this assumption, as it shows a resilin-dominated area basally (sbsp in Fig. 4a, b), comparable to the discoidal spine(s) described for praying 
mantises. The distal face of the subbasal spine, the ventral surface, as well as the lateral base appear to be highly sclerotized (Fig. 4b, e). The medial and posteromedial face, as well as the median base and the following ventral area of the femur in contrast, appear to be resilin-dominated (Fig. 4b, e). This indicates a similar mechanism-a lever and ratchet system-formed by the elongated sickle-shape of tibia and tarsus in M. styriaca, resembling what is described for praying mantises by Loxton and Nicholls (1979). The anteroventral carina with its spines seems to work as a unit, with different possible functions. On the one hand, the strongly sclerotized surface, in interaction with the sclerotized cutting edge of the ventral tibia (ce in Fig. 4d), might work in a similar way as the carnassial teeth in carnivorous Mammalia (Mellett 1981). This mechanism could be used to get a hold on the prey, but also maybe breaking its outer cuticle, similar to the mentioned carnassial's. On the other hand, it could fix the spatial orientation of the tibia, ensuring its grip on the prey. In this case, the subbasal spine, in combination with a notch located anteroventral at the distal end of the femur, prevents the tibia from moving anteriorly. Additionally, the posteroventral carina with the anteroventral spines prevent the tibia from moving posteriorly (Fig. 3a, b). The subbasal spine, described here in detail for M. styriaca (Figs. 3, 4a, b), is not present in all extant Mantispidae (see Plega signata (Hagen, 1877) in Ferris 1940), but well known from the fossil record of, for example, Depranicinae (Shi et al. 2015; Pérez-de la Fuente and Peñalver 2019). As elaborately described by Shi and colleagues (2020) this spine seems to be a prominent character of the group comprising Depranicinae, Calomantispinae and Mantispinare, a well-established sister group relationship within Mantispidae (Liu et al. 2014).

Different setups of spines on the sub-chelate type raptorial forelegs are also present in the hemipteran group Reduviidae (Weirauch et al. 2011; Zhang et al. 2016; CastroHuertas et al. 2019), including enlarged spines that might resemble the here, and in mantises (Loxton and Nicholls 1979), described system.

Outside Insecta, enlarged spines to capture prey with subchelate type raptorial forelegs are well-known from mantis shrimps (Crustacea: Stomatopoda). These spines are most likely strongly sclerotised and immobile because they are used to spear prey (Caldwell and Dingle 1975; deVries et al. 2012).

The mentioned sickle-shaped tibia of $M$. styriaca seems to be present in Mantispidae in general (Ferris 1940; Shi et al. 2015, 2020; Pérez-de la Fuente and Peñalver 2019). In M. styriaca no tibial spines are present; in general, no to minute spines on the tibial surface are present in Mantispidae (Pérez-de la Fuente and Peñalver 2019). This tibial condition differs significantly from the one found in Reduviidae (Weirauch et al. 2011; Zhang et al. 2016; Castro-Huertas et al. 2019) and Mantodea (Roy 1999; Wieland 2013 or
Brannoch et al. 2017), both groups showing a significant density of spines on both the femur and the tibia. In Belostomatidae and Nepidae (Insecta: Hemiptera), however, subchelate type raptorial forelegs show no spines at all, neither on the tibia nor the femur (Schuh and Slater 1995).

The muscle set-up and movement sequence of the femur and tibia are of particular interest. The thin and long muscle M25 is running dorsally from the most proximal region of the femur to the proximal tibial rim, enabling the opening of the tibia - abduction (Fig. 5e, 6). Its large antagonists M24a and M24b, are capable of a fast and powerful closing of the tibia-adduction (Figs. 5a, e, 6). Together, these latter two muscles cover approximately $2 / 3$ of the inner surface of the femur (Fig. 5a, e), converging at the distal end of the femur into a shared tendon. This tendon separates in two independent tendons at its apical end, attaching to the anterodorsal and posterodorsal proximal inner surface of the widened tibial base. Furthermore, muscle M $24 \mathrm{c}$ originates at the lateral and median side of the ventral inner surface of the widened tibial base (Figs. 5a, 6), attaching to the mentioned tendon.

In combination with two cuticular protrusions (dorsotibial protrusion—dtp and ventrotibial complex — vtc), these four muscles likely represent a latch-mediated spring-actuated mechanism (Longo et al. 2019). This catapult mechanism is proposed here for mantis lacewings for the first time. Catapult systems are well known to operate ultra-fast predatory strikes, for example in dragonfly larvae (Büsse et al. 2021), or mantis shrimps (Patek et al. 2004). Here, it is functioning comparable to the described catapult systems in cicadas (Gorb 2004) or trap-jaw ants (Gronenberg 1999). Figure 6 shows a functional schematic of the hypothetical action of all structures involved in the process. At first, muscle M25 (blue) contracts, applying force to the dorsoproximal tibial rim, opening the tibia (Fig. 6a). Simultaneously, the 'dorsotibial protrusion' (dtp in Fig. 6b) deflects the tendon (green) in ventral direction, while the 'ventrotibial complex' (vtc in Fig. 6b) - this triangular sclerite is shaped like the collar bone (clavicle) of birds-deflects the tendon in dorsal direction (Fig. 6b). The tendon is deformed-absorbing kinetic energy, by the contraction of muscles M24a and M24b (Fig. 6b, d, e). Simultaneously, the shape of the ventrotibial complex becomes important; this triangular sclerite shows an anterior and posterior protrusion with a median gap proximally (Fig. 6d, e). To allow for spring actuation, the trigger muscle M24c contracts, pulling the tendon in lateral direction (Fig. 6b, e). At this point, the system is fully loaded and ready to strike, with the trigger muscle M24c in contracted state (Fig. 6b, e). To launch the spring, the trigger muscle M24c relaxes, causing the tendon to slide in ventromedial direction (Fig. 6c, d). Thereby, the two parts of the tendon are pulled over the edge of the anterior and posterior protrusion into the median gap of the ventrotibial complex (Fig. 6c, d). By crossing the axis of rotation, the tendon 


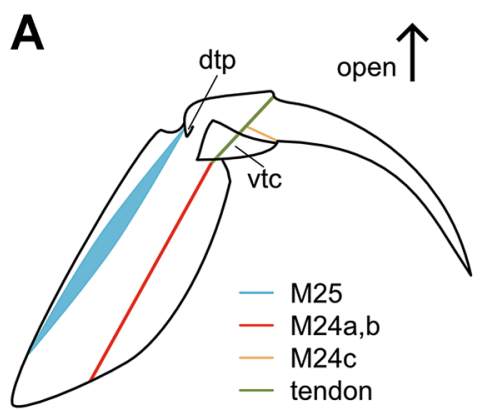

D

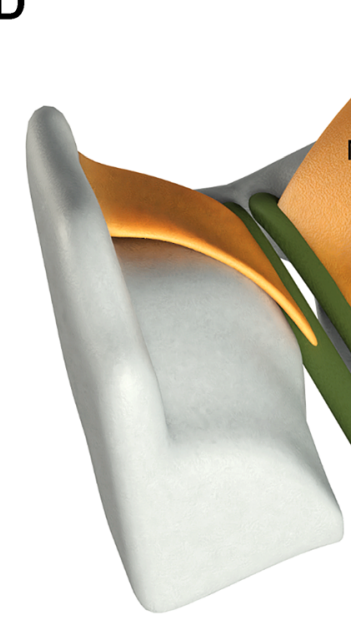

relaxed muscle
B

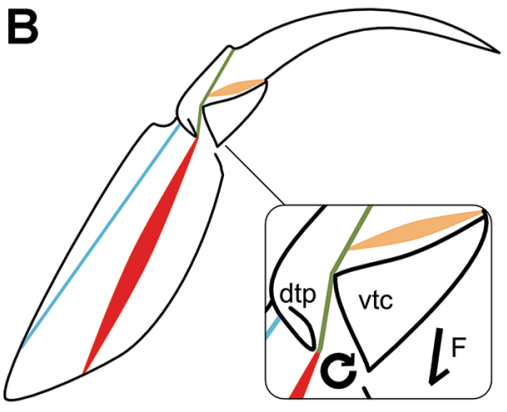

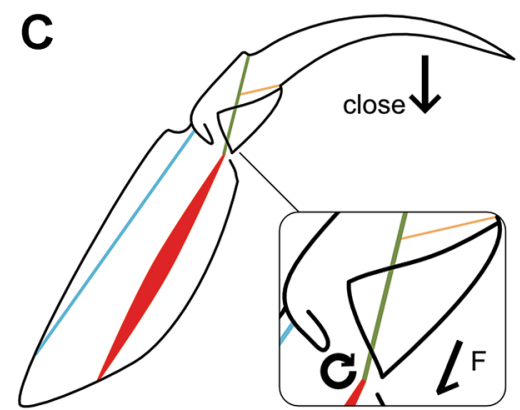

$\mathbf{E}$

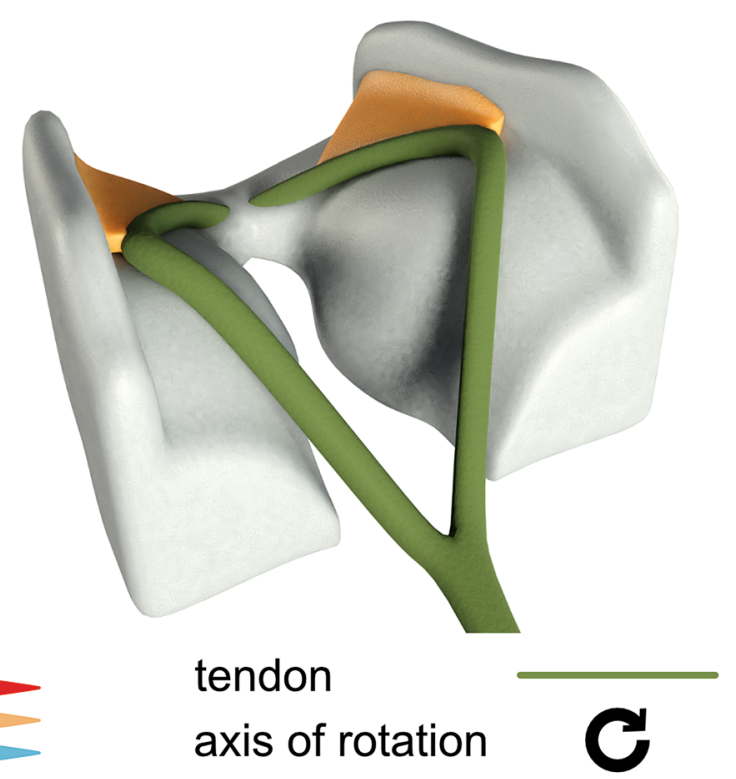

Fig. 6 Functional schematic of the tibial catapult mechanism. a opening of the tibia; $\mathbf{b}$ loading of the catapult mechanism; $\mathbf{c}$ releasing of the catapult mechanism; $\mathbf{d}$, e 3D schematic of the hypothetical mus-

now returns into its original shape, releasing the previously stored energy and causing the fast-closing movement of the tibia (Fig. 6c). The video footage supports our morphological findings. When catching prey, the tibia of $M$. styriaca is always fully opened, before movements of the remaining parts of the predatory foreleg takes place-representing the loading of the catapult mechanism (Granier 2019: 1:27).

\section{Conclusion}

Using $\mu \mathrm{CT}$ analysis, we could confirm all previously described muscles for mantis lacewings, including one important new muscle-M24c. Considering the intrinsic musculature, the muscle setup in adult $M$. styriaca is different to, for example, previous descriptions for praying mantises. Additionally, we revealed a key feature of the raptorial forelegs in $M$. styriaca, previously only known cle tendon complex of the catapult mechanism. d catapult unloaded; e catapult loaded. Dtp dorsal tibial protrusion, $M$ muscle, Vtc ventral tibial complex for praying mantises: the subbasal spine in collaboration with the elongated sickle shape, formed by the tibia together with the tarsus, working as a lever and ratchet system, which is supported by the material properties indicated by our CLSM analysis. Furthermore, we proposed a latch-mediated spring-actuated (catapult) mechanism for the closing process of the tibia on the femur, unknown for mantis lacewings before. Our investigation resulted in new insights into the morphology of the raptorial forelegs and the functional mechanism of the predatory strike in mantis lacewings. Nevertheless, our study is only a door opener, to prove the proposed catapult mechanism in $M$. styriaca experimentally.

Acknowledgements We are grateful for the support by the members of the Functional Morphology and Biomechanics Group at Kiel University, especially to T. H. Büscher, L. M. Hindenberg and A. Koehnsen. Many thanks to S. Randolf, Natural History Museum Vienna, Austria for providing specimens. 
Author contributions SB and SNG designed the project and developed the concept of the study. FB and SB did the $\mu \mathrm{CT}$ analysis and postprocessing. SB carried out the CLSM analysis. All authors wrote the manuscript as well as read and approved the final version.

Funding Open Access funding enabled and organized by Projekt DEAL. SB is directly supported through the DFG grants BU3169/1-1 \& 1-2.

Data availability All data supporting our findings are presented in the paper and the supplementary material respectively. The raw data of the CT- and CLSM-analysis can be made available on reasonable request.

\section{Declarations}

Conflict of interest We declare that we have no known competing interests.

Consent to participate and publication We all agree(ed).

Open Access This article is licensed under a Creative Commons Attribution 4.0 International License, which permits use, sharing, adaptation, distribution and reproduction in any medium or format, as long as you give appropriate credit to the original author(s) and the source, provide a link to the Creative Commons licence, and indicate if changes were made. The images or other third party material in this article are included in the article's Creative Commons licence, unless indicated otherwise in a credit line to the material. If material is not included in the article's Creative Commons licence and your intended use is not permitted by statutory regulation or exceeds the permitted use, you will need to obtain permission directly from the copyright holder. To view a copy of this licence, visit http://creativecommons.org/licenses/by/4.0/.

\section{References}

Andersen SO (1979) Biochemistry of insect cuticle. Ann Rev Entomol 24:29-61. https://doi.org/10.1146/annurev.en.24.010179.000333

Appel E, Gorb SN (2011) Resilin-bearing wing vein joints in the dragonfly Epiophlebia superstes. Bioinspir Biomim 6:046006

Aspöck U, Aspöck H (1994) Zur Nomenklatur der Mantispiden Europas (Insecta: Neuroptera:Mantispidae). Annal Naturhist Mus Wien 96B:99-114

Aspöck U, Aspöck H (2007) Verbliebene Vielfalt vergangener Blüte. Zur Evolution, Phylogenie und Biodiversität der Neuropterida (Insecta: Endopterygota). Denisia 20:451-516

Aspöck H, Aspöck U, Hölzel H (1980) Die Neuropteren Europas. Eine zusam- menfassende Darstellung der Systematik, Ökologie und Chorologie der Neuropteroidea (Megaloptera, Raphidioptera, Planipennia) Europas. Goecke \& Evers, Krefeld

Bäumler F, Büsse S (2019) Resilin in the flight apparatus of Odonata (Insecta) — cap tendons and their biomechanical importance for flight. Biol Lett 15:20190127. https://doi.org/10.1098/rsbl.2019.0127

Bäumler F, Koehnsen A, Tramsen HT, Gorb SN, Büsse S (2020) Illuminating nature's beauty: modular, scalable and low-cost LED dome illumination system using 3D-printing technology. Sci Rep 10:12172

Beutel RG, Friedrich F, Ge S-Q, Yang X-K (2014) Insect morphology and phylogeny. De Gruyter Graduate, Berlin/Boston

Boyden TC (1983) Mimicry, predation and potential pollination by the Mantispid, Climaciella brunnea var. instabilis (Say) (Mantispidae: Neuroptera). J New York Entomol Soc 91:508-511

Brannoch SK, Wieland F, Rivera J, Klass K-D, Béthoux O, Svenson GJ (2017) Manual of praying mantis morphology, nomenclature, and practices (Insecta, Mantodea). ZooKeys 696:1-100. https:// doi.org/10.3897/zookeys.696.12542

Brémond J (1974) Remarques sur le phenomene du convergence des membres prehensiles chez la Mante religieuse et le Crustace' Squilla mantis. Entomologiste 30:183-188

Büsse S, Gorb SN (2018) Material composition of the mouthpart cuticle in a damselfly larva (Insecta: Odonata) and its biomechanical significance. R Soc Open Sci 5:172117

Büsse S, Hörnschemeyer T, Hohu K, McMillan DM, Edgerly JS (2015) The spinning apparatus of webspinners-functional-morphology, morphometrics and spinning behaviour. Sci Rep 5:9986. https:// doi.org/10.1038/srep09986

Büsse S, Büscher TH, Kelly ET, Heepe L, Edgerly JS, Gorb SN (2019a) Pressure-induced silk spinning mechanism in webspinners (Insecta: Embioptera). J Soft Matter 15:9742

Büsse S, Büscher TH, Heepe L, Gorb SN (2019b) Adaptations of dragonfly larvae and their exuviae (Insecta: Odonata), attachment devices and their crucial role during emergence. J Insect Physiol 117:103914

Büsse S, Koehnsen A, Rajabi H, Gorb SN (2021) A controllable dual-catapult system inspired by the biomechanics of the dragonfly larvae's predatory strike. Sci Robot 6:eabc8170. https:// doi.org/10.1126/scirobotics.abc8170

Caldwell RL, Dingle H (1975) Ecology and evolution of agonistic behavior in stomatopods. Naturwissenschaften 62:214-222

Castro-Huertas V, Forero D, Grazia J (2019) Comparative morphology of the raptorial leg in thread-legged bugs of the tribe Metapterini Stål, 1859 (Hemiptera, Heteroptera, Reduviidae, Emesinae). Zoomorphology 138:97-116. https://doi.org/10. 1007/s00435-019-00431-x

deVries MS, Murphy EAK, Patek SN (2012) Strike mechanics of an ambush predator: the spearing mantis shrimp. J Exp Biol 215:4374-4384. https://doi.org/10.1242/jeb.075317

Dittmann IL, Hörnig MK, Haug JT, Haug C (2015) Raptoblatta waddingtonae $\mathrm{n}$. gen. et $\mathrm{n}$. sp.- an Early Cretaceous roach-like insect with a mantodean-type raptorial foreleg. Palaeodiversity 8:103-111

Ferris GF (1940) The Morphology of Plega signata (Hagen) (Neuroptera: Mantispidae). Microentomology 5:33-56

Frantsevich L (1998) The coxal articulation of the insect striking leg: a comparative study. J Morph 236:127-138. https://doi.org/10. 1002/(SICI)1097-4687(199805)236:2\%3c127::AID-JMOR4\% 3e3.0.CO;2-2

Friedrich F, Beutel RG (2008) The thorax of Zorotypus (Hexapoda, Zoraptera) and a new nomenclature for the musculature of Neoptera. Arthropod Struct Dev 37:29-54. https://doi.org/10. 1016/j.asd.2007.04.003

Gorb SN (1999) Serial elastic elements in the damselfly wing: mobile vein joints contain resilin. Naturwissenschaften $86: 552-555$. https://doi.org/10.1007/s001140050674

Gorb SN (2004) The jumping mechanism of cicada Cercopis vulnerata (Auchenorrhyncha, Cercopidae): skeleton-muscle organisation, frictional surfaces, and inverse-kinematic model of leg movements. Arthropod Struct Dev 33:201-220. https://doi.org/ 10.1016/j.asd.2004.05.008

Granier, C. [yackphoto], (11. 08. 2019) Mantispa styriaca 1920x1080 Qf. https://www.youtube.com/watch?v=hQTSr LUSqGo. Accessed 16 Mar 2020

Gray PT, Mill PJ (1983) The mechanics of the predatory strike of the praying mantis Hierodula membranacea. J Exp Biol 107:245-275

Gray PT, Mill PJ (1985) The musculature of the prothoracic legs and its innervation in Hierodula membranacea (Mantidea). Philos Trans R Soc Lond B 309:479-503. https://doi.org/10.1098/rstb. 1985.0094

Gronenberg SJW (1999) The control of mandible movements in the ant Odontomachus. J Insect Physiol 45:231-240 
Haas F, Gorb SN, Wootton RJ (2000) Elastic joints in dermapteran hind wings: materials and wing folding. Arthropod Struct Dev 29:137-146. https://doi.org/10.1016/S1467-8039(00)00025-6

Haug JT, Müller P, Haug C (2018) The ride of the parasite: a 100-million-year old mantis lacewing larva captured while mounting its spider host. Zoological Lett 4:31. https://doi.org/10.1186/ s40851-018-0116-9

Hörnig MK, Haug JT, Haug C (2017) An exceptionally preserved 110 million years old praying mantis provides new insights into the predatory behaviour of early mantodeans. PeerJ 5:e3605. https:// doi.org/10.7717/peerj.3605

Jandausch K, Beutel R, Pohl H, Gorb SN, Büsse S (2018) The legs of "spider associated" parasitic primary larvae of Mantispa aphavexelte (Mantispidae, Neuroptera)—attachment devices and phylogenetic implications. Arthropod Struct Dev 47:449-456. https:// doi.org/10.1016/j.asd.2018.06.002

Jo Alwood (10. 10. 2016) Praying Mantis catching prey CLOSEUPSNARRATED. https://www.youtube.com/watch? $\mathrm{v}=\mathrm{mSuLdBVS}-$ naM. Accessed 16 Mar 2020

Josephs B (25. 10. 2017) Preying mantis vs fly. https://www.youtube. $\mathrm{com} /$ watch?v=NB6-gG05UdU. Accessed 16 Mar 2020

Kaston BJ (1938) Mantispidae parasitic on spider egg sacs. J New York Entomol Soc 46:147-153

Lequet A (30. 07. 2018) La Mantispe de Styrie (Mantispa styriaca), « scènes de vie », par André Lequet. https://www.youtube.com/ watch?v=ByeWIMpdang. Accessed 16 Mar 2020

Liu X, Winterton SL, Wu C, Piper R, Ohl M (2014) A new genus of mantidflies discovered in the Oriental region, with a higher-level phylogeny of Mantispidae (Neuroptera) using DNA sequences and morphology. Syst Entomol 40:183-206. https://doi.org/10. 1111/syen. 12096

Longo SJ, Cox SM, Azizi E, Ilton M, Olberding JP, StPierre R, Patek SN (2019) Beyond power amplification: latch-mediated spring actuation is an emerging framework for the study of diverse elastic systems. $\mathbf{J}$ Exp Bio. 222:jeb197889. https://doi.org/10.1242/jeb.197889

Loxton RG, Nicholls I (1979) The functional morphology of the praying mantis forelimb (Dictyoptera: Mantodea). Zool J Linn Soc 66:185-203. https://doi.org/10.1111/j.1096-3642.1979.tb01908.x

Martins AC, Meloand GAR, Renner SS (2014) The corbiculate bees arose from New World oil-collecting bees: implications for the origin of pollen baskets. Mol Phylogenet Evol 80:88-94. https:// doi.org/10.1016/j.ympev.2014.07.003

Matsuda R (1970) Studies on the thoracic musculature in insects. Mem Fac Sci Taihoku Imp Univ 24:1-343

Mellett JS (1981) Mammalian carnassial function and the "Every Effect." J Mamm 62:164-166. https://doi.org/10.2307/1380488

Michels J, Gorb SN (2012) Detailed three-dimensional visualization of resilin in the exoskeleton of arthropods using confocal laser scanning microscopy. J Microsc 245:1-16. https://doi.org/10.1111/j. 1365-2818.2011.03523.x

Patek SN, Korff WL, Caldwell RL (2004) Biomechanics: deadly strike mechanism of a mantis shrimp. Nature 428:819-820. https://doi. org/10.1038/428819a

Peisker H, Michels J, Gorb SN (2013) Evidence for a material gradient in the adhesive tarsal setae of the ladybird beetle Coccinella septempunctata. Nat Comm 4:1661. https://doi.org/10.1038/ncomm s2576

Pérez-de la Fuente R, Peñalver E (2019) A mantidfly in Cretaceous Spanish amber provides insights into the evolution of integumentary specialisations on the raptorial foreleg. Sci Rep 9:13248. https://doi.org/10.1038/s41598-019-49398-1

Petersen DS, Kreuter N, Heepe L, Büsse S, Wellbrock AHJ, Witte C, Gorb SN (2018) Holding tight to feathers-structural specializations and attachment properties of the avian ectoparasite Crataerina pallida (Diptera, Hippoboscidae). J Exp Biol 221:179242. https://doi.org/10.1242/jeb.179242
Pohl H (2010) A scanning electron microscopy specimen holder for viewing different angles of a single specimen. Microsc Res Techniq 73:1073-1076

Poivre C (1976) Observations sur la biologie, le comportement et le phénomène de convergence chez les Mantispidés [Planipennes]. Entomologiste 32:2-19

Rajabi H, Shafiei A, Darvizeh A, Gorb SN (2016) Resilin microjoints: a smart design strategy to avoid failure in dragonfly wings. Sci Rep 6:39039. https://doi.org/10.1038/srep39039

Redborg KE (1998) Biology of the Mantispidae. AnnRev Entomol 43:175-194. https://doi.org/10.1146/annurev.ento.43.1.175

Redborg KE, MacLeod EG (1983) Climaciella brunnea (Neuroptera: Mantispidae): a mantispid that obligately boards spiders. J Nat Hist 17:63-73

Rice ME (1986) Communal oviposition by Mantispa fuscicornis (Say) (Neuroptera: Mantispidae) and subsequent larval parasitism on spiders (Arachnida: Araneida) in South Texas. J Kansas Entomol Soc 59:121-126

Roy R (1999) Morphology and taxonomy in the praying mantids. In: Prete FR, Wells H, Wells PH, Hurd LH (Eds). Johns Hopkins University Press, Baltimore, US

Schuh RT, Slater JA (1995) True Bugs of the World (Hemiptera Heteroptera): Classification and Natural History. Cornell University Press, Ithaca, NY, USA

Shi C, Ohl M, Wunderlich J, Ren D (2015) A remarkable new genus of Mantispidae (Insecta, Neuroptera) from Cretaceous amber of Myanmar and its implications on raptorial foreleg evolution in Mantispidae. Cretac Res 52:416-422. https://doi.org/10.1016/j. cretres.2014.04.003

Shi C, Yang Q, Winterton SL, Pang H, Ren D (2020) Stem-Group fossils of Symphrasinae shed light on eraly evolution of Mantispidae (Insecta, Neuroptera). Papers Palaeontol 6:143-154. https://doi. org/10.1002/spp2.1265

Simpson KW (1975) Biology and immature stages of three species of Nearctic Ochthera (Diptera: Ephydridae). Proc Entomol Soc Washington 77:129-155

Ulrich H (1965) Der Fang- und Greifapparat von Mantispa - ein Vergleich mit Mantis. Nat Mus 95:499-508

Vincent JFV (2002) Arthropod cuticle: a natural composite shell system. Compos Part A 33:1311e1315. https://doi.org/10.1016/ S1359-835X(02)00167-7

Weirauch C, Forero D, Jacobs DH (2011) On the evolution of raptorial legs - an insect example (Hemiptera: Reduviidae: Phymatinae). Cladistics 27:138-149. https://doi.org/10.1111/j.1096-0031.2010. 00325.x

Wieland F (2013) The phylogenetic system of Mantodea (Insecta: Dictyoptera). Spec Phylo Evol 3:3-222. https://doi.org/10.17875/ gup2013-711

Willkommen J, Michels J, Gorb SN (2015) Functional morphology of the male caudal appendages of the damselfly Ischnura elegans (Zygoptera: Coenagrionidae). Arthropod Struct Dev 44:289-300. https://doi.org/10.1016/j.asd.2015.04.002

Willmann R (1990) The phylogenetic position of the Rhachiberothinae and the basal sister-group relationships within the Mantispidae (Neuroptera). Syst Entomol 15:253-265. https://doi.org/10.1111/j. 1365-3113.1990.tb00316.x

Zhang J, Gordon ERL, Forthman M, Hwang WS, Walden K, Swanson DR, Johnson KP, Meier R, Weirauch C (2016) Evolution of the assassin's arms: insights from a phylogeny of combined transcriptomic and ribosomal DNA data (Heteroptera: Reduvioidea). Scie Rep 6:22177. https://doi.org/10.1038/srep22177

Publisher's Note Springer Nature remains neutral with regard to jurisdictional claims in published maps and institutional affiliations. 